\title{
BMJ Open Health-related quality-of-life of coal- based sponge iron plant workers in Barjora, India: a cross-sectional study
}

\author{
Kaushik Chattopadhyay, ${ }^{1}$ Chaitali Chattopadhyay, ${ }^{2}$ Eva Kaltenthaler ${ }^{3}$
}

To cite: Chattopadhyay K, Chattopadhyay $\mathrm{C}$,

Kaltenthaler E. Health-related quality-of-life of coal-based sponge iron plant workers in Barjora, India: a crosssectional study. BMJ Open 2014;4:e006047.

doi:10.1136/bmjopen-2014006047

- Prepublication history for this paper is available online. To view these files please visit the journal online (http://dx.doi.org/10.1136/ bmjopen-2014-006047)

Received 5 July 2014 Revised 5 August 2014 Accepted 13 August 2014

CrossMark

${ }^{1}$ Faculty of Epidemiology and Population Health, London School of Hygiene and Tropical Medicine, London, UK

${ }^{2}$ Monitoring and Evaluation, United Nations Office for Project Services, Geneva, Switzerland

${ }^{3}$ Health Economics and Decision Science, School of Health and Related Research, The University of Sheffield, Sheffield, UK

\section{Correspondence to} Dr Kaushik Chattopadhyay; Kaushik.Chattopadhyay@ Ishtm.ac.uk

\section{ABSTRACT \\ Objectives: During the last decade, coal-based sponge iron plants, a highly polluted industry, have grown rapidly in Barjora, India. Understanding their workers' perception of health is essential in people-centered healthcare. The aim of the study was to assess their health-related quality- of-life (HRQoL), and to determine factors that independently predict their $\mathrm{HRQOL}$. \\ Design: Cross-sectional study. \\ Setting: Coal-based sponge iron plants in Barjora, India. Participants: 258 coal-based sponge iron plant workers. Primary outcome measure: $\mathrm{HRQOL}$ was measured using the EuroQol-5D-5L.}

Results: The response rate was $100 \%$. Participants with problems in mobility, self-care, usual activities, pain/ discomfort and anxiety/depression were $23.3 \%, 5.1 \%$, $10.9 \%, 39.5 \%$ and $45.5 \%$, respectively. $36.8 \%$ of participants reported health state 11111 (no problem in any EQ-5D dimension). The mean visual analogue scale (EQ-VAS) was 69.8 (18.5 SD). The odds of mobility problems decreased with age $(\mathrm{OR} 0.95,95 \% \mathrm{Cl} 0.91$ to $0.99, \mathrm{p}=0.016$ ), were lower in participants with presence/ history of any respiratory disease $(0.27,0.13$ to 0.55 , $\mathrm{p}<0.001)$, scheduled caste/scheduled tribe/other backward class workers $(0.44,0.22$ to $0.89, p=0.021)$, manual workers $(0.40,0.16$ to $0.99, p=0.047)$ and nonsmokers $(2.63,1.27$ to $5.46, p=0.009)$. The odds of pain/ discomfort and anxiety/depression were lower in participants with any respiratory disease $(0.44,0.24$ to $0.79, p=0.006$; and $0.52,0.29$ to $0.92, p=0.026$, respectively). The EQ-VAS was worse in manual participants (coefficient $-6.91,95 \% \mathrm{Cl}-12.40$ to -1.41 , $\mathrm{p}=0.014)$, with any respiratory disease $(-8.13,-13.12$ to $-3.13, p=0.002)$, alcohol drinkers $(-4.81,-9.47$ to $-0.15, p=0.043)$, literates $(7.70,0.97$ to $14.43, p=0.025)$ and Hindus (13.41, 2.62 to $24.20, p=0.015)$.

Conclusions: Many coal-based sponge iron plant workers in Barjora have problems in their HRQoL, and the predictors of different aspects of HRQoL were identified. The study findings could be taken into consideration in future interventional studies aimed at improving the $\mathrm{HRQ}$ oL of these workers.

\section{INTRODUCTION}

Sponge iron or direct reduced iron (DRI) is a transitional material used in the

\section{Strengths and limitations of this study}

- This is the first study to explore health-related quality-of-life (HRQoL) of coal-based sponge iron plant workers.

- HRQoL measurement is subjective to participants and thus, a valid and reliable tool (EQ-5D-5L) was used.

- The response rate was $100 \%$. Missing data could lead to bias, but it was extremely low in this study.

- Participants who were absent from work on the dates of the survey were excluded, and this absence from work could be due to poor HRQoL which could have underestimated the prevalence of poor HRQoL.

- As this was a cross-sectional study, it was not possible to determine the causal association between different variables and HRQoL.

production of steel. Either coal or natural gas is used in sponge iron production. In India, non-coking coal is easily available. Thus, the sector depends mostly on coalbased sponge iron and nearly $80 \%$ of the total coal-based sponge iron plants are located in India. ${ }^{1}$ About $60 \%$ of this production comes from small-scale industries in the unorganised sector with poor pollution control facilities. ${ }^{1}$ During the past decade, these sponge iron plants have rapidly grown in the Barjora block of Bankura district, a deprived district in West Bengal. ${ }^{2}$ These factories are categorised as red industries (highly polluted industries) and the major pollutants are of three types: solid waste heavy metals (cadmium, chromium, lead, mercury and nickel); particulate matters (suspended particulate matter and respirable particulate matter) and gaseous pollutants (oxides of sulfur and nitrogen, and hydrocarbons) ${ }^{1-4}$ The toxic effects of solid waste heavy metals are varied and often take several years to manifest. However, the toxic effects of particulate matters and gaseous pollutants are often rapid and include 
respiratory diseases (cough, phlegm, bronchitis, asthma and allergy). ${ }^{1-4}$ The importance of prevention and treatment of these ailments is well acknowledged, however, understanding workers' perception of health is also essential in people-centered healthcare. ${ }^{5}{ }^{6}$ Healthrelated quality-of-life (HRQoL) is a complex and multidimensional concept of the well-being of a person and their perception of health. ${ }^{7}$ HRQoL is one of the essential aspects of health, which is embedded in the physical, mental, social and cultural context. ${ }^{7}$ Poor HRQoL places a burden on the individual, family, community and health services and thus, HRQoL is of major public health importance. ${ }^{5}{ }^{6}$ Research has been conducted to explore HRQoL of the general population and other workforce groups in various countries, ${ }^{8-14}$ but none among coal-based sponge iron plant workers. The aim of the study was to assess their HRQoL and to determine factors that independently predict their HRQoL. Knowledge of factors associated with HRQoL of these workers would provide valuable information about strategies that professionals and providers of healthcare can address to improve their HRQoL.

\section{METHODS}

Study design, participants, area and inclusion/exclusion criteria

A cross-sectional study was conducted among coal-based sponge iron plant workers in Barjora block (Bankura district, West Bengal, India) as shown in figure 1. Participants who gave written informed consent to participate in the study were included, and those who were absent from work on the dates of the survey were excluded.

\section{Data collection procedure and tool}

A survey was conducted with a quantitative questionnaire (available in English, Bengali and Hindi languages) in May and June 2013. The questionnaire was either selfcompleted by the participant (if literate) or was completed by the field worker (for illiterate participants). In the latter case, each question was shown and read to the participant who was asked to say and point out the answer. Each session lasted for about $30 \mathrm{~min}$ and was completed in one sitting. The data were entered on the day of its collection.

Section I of the questionnaire included the following variables: age (in years), sex, mother tongue (proxy measures for local ethnic origin, culture and lifestyle), religion, social caste (general or scheduled caste (SC)/ scheduled tribe (ST)/other backward class (OBC)), marriage, education (literate or illiterate), work type (non-manual or manual), working hours per day (8 (normal) $)^{15}$ or more than 8 ), salary per month (5395 Indian rupees (INR: minimum monthly wage of an unskilled worker) ${ }^{15}$ or more, or less than 5395 INR), total duration of work in this type of factory (proxy measure for exposure time), smoking, smokeless tobacco intake, alcohol drinking, house type (pucca/ semipucca (at least some high-quality construction materials such as bricks, tiles, cement and concrete) or kachcha (low-quality construction materials such as mud and thatch) $),{ }^{16}$ people living in a room (less than 3 , or 3 or more to indicate crowding), ${ }^{16}$ presence/history of any respiratory disease and health insurance. All the variables were dichotomous except age and total duration of work in this type of factory. Section I was designed in English, translated into Bengali and Hindi, reviewed by a local primary school teacher and pretested extensively on six local similar workers who were not involved in the study. Section II included the standardised EuroQol-5D-5L (EQ-5D-5L) questionnaire to measure the generic HRQoL (time-recall: at the time of completion). ${ }^{17}$ This questionnaire has been widely used in the general population, different workforce groups and patients. ${ }^{8-11} 17$ The descriptive system comprises five dimensions: mobility, self-care, usual activities, pain/discomfort and anxiety/depression. Each dimension has five levels: no problems, slight problems, moderate problems, severe problems and extreme problems. The EQ visual analogue scale (EQ-VAS) reports a participant's self-rated health on a $20 \mathrm{~cm}$ vertical scale. This scale is numbered from 0 (worst possible health) to 100 (best possible health). The EQ-5D-5L is available in English, Bengali and Hindi. ${ }^{17}$ All cut-off points to determine poor outcomes were predefined.

\section{Sample size}

As this was the first study of HRQoL of coal-based sponge iron plant workers, no information was available on which to base the sample size calculation. Instead, a webbased sample size calculator, Creative Research Systems, ${ }^{18}$ was used to calculate the sample size, using the following assumptions/information: confidence level (95\%), margin of error $(5 \%)$ and population size (662 information from the factory directors in Barjora). A random sample of 258 such workers was required, assuming a response rate of $95 \%$. A numbered list of all 662 workers was created (worker \#1, worker \#2, worker \#3, and so forth). A web-based randomiser, Research Randomizer, ${ }^{19}$ was used to generate 1 set of 258 unique, sorted numbers with a range from 1 to 662 (representing the workers' assigned numbers).

\section{Ethics}

Approval was received from the Barjora Block Development Office Committee (the committee was based on the Indian Council of Medical Research Ethical Guidelines for Biomedical Research on Human Participants, $2006^{20}$ ). Information sheets and consent forms were available in English, Bengali and Hindi. The study objectives were explained to all the eligible participants and written informed consent was taken from those interested in participating. Illiterate participants were requested to put their left hand thumb impression on the consent form. Participants were not compelled 


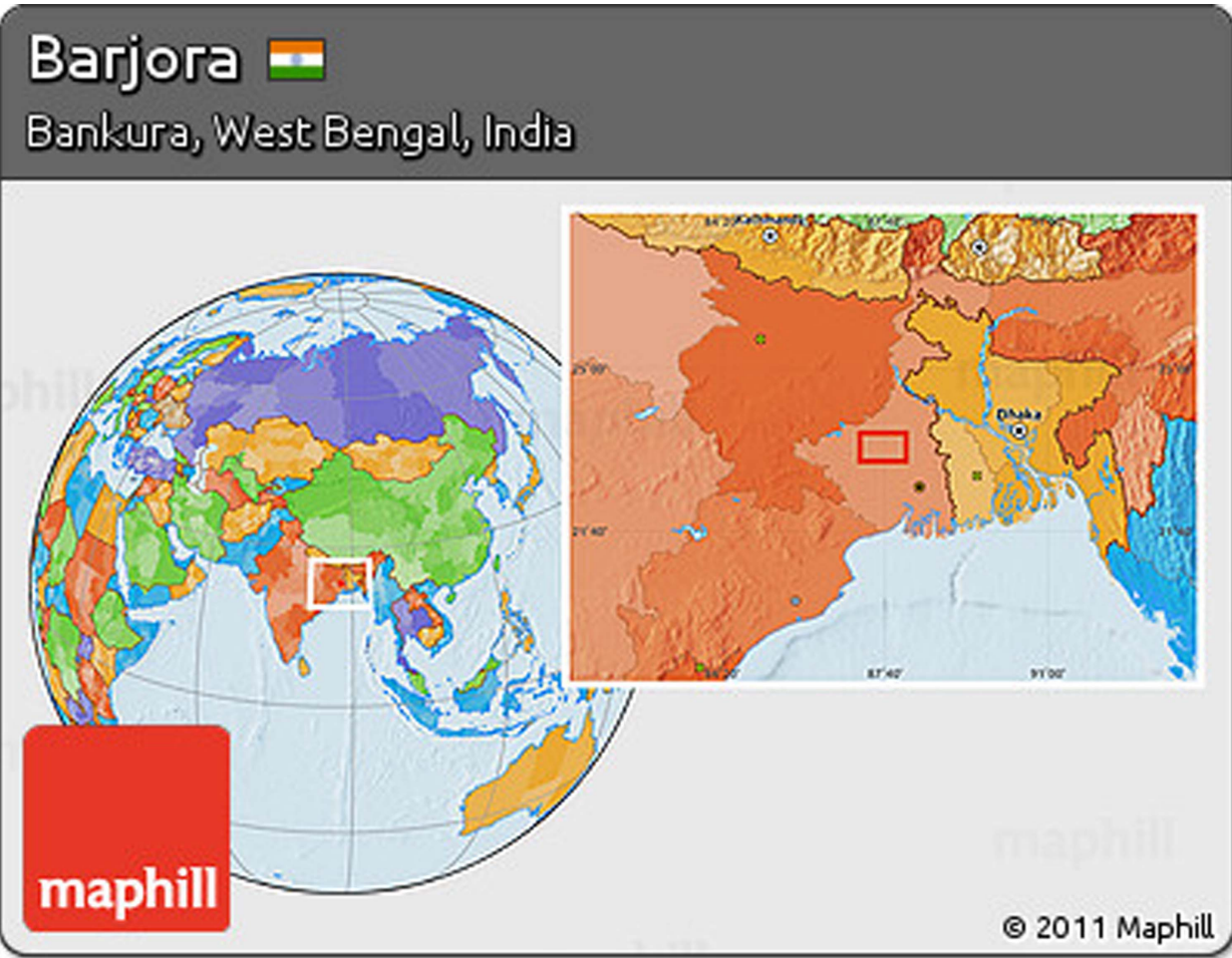

Figure 1 Location of Barjora, India.

and were free to participate in the study. They were assured regarding the anonymity, privacy, confidentiality and data protection of their information.

\section{Statistical analyses}

For the purpose of analysis, the EQ-5D-5L dimensions was categorised into: 'no problems' (level 1) and 'problems' (level 2 to 5), ${ }^{17}$ and numbers and proportions were calculated being a categorical variable. The internal consistency reliability of EQ-5D dimensions was estimated using Cronbach's $\alpha$. The EQ-VAS is a continuous variable and was normally distributed, thus mean and SD were calculated. Appropriate methods were used to investigate the association between the EQ-5D dimensions/EQ-VAS and Section I variables $\left(\chi^{2}\right.$ test, Fisher's exact test, sample t-test or simple logistic/linear regression). To identify any independent association, corresponding multiple logistic and linear regression models were developed using the backward stepwise regression analysis. All the Section I variables were included. Multiple regression models included a sample with unknown values for these adjusted variables. OR or coefficients and their respective 95\% CI were calculated. The results were considered significant when $p$ values were less than or equal to 0.05 . All data were analysed using STATA V.12 for Windows software. ${ }^{21}$

\section{RESULTS}

The response rate was $100 \%$. All the participants were men with a mean age of 35.4 years. The percentage of participants with problems in mobility, self-care, usual activities, pain/discomfort and anxiety/depression were $23.3 \%, 5.1 \%, 10.9 \%, 39.5 \%$ and $45.5 \%$, respectively. Health state 11111 (no problem in any EQ-5D dimension indicating good health) was reported by $36.8 \%$ of participants. The Cronbach's $\alpha$ of EQ-5D dimensions was 0.75 . The mean EQ-VAS was 69.8 (18.5 SD). Table 1 reports the characteristics of coal-based sponge-iron factory workers with no problem and problems in the EQ-5D dimensions and EQ-VAS. Marriage $(p=0.031)$, working hours/day $(p=0.020)$, total duration of work in this type of factory $(p=0.031)$ and presence/history of any respiratory disease $(p<0.001)$ were found to be associated with mobility. Respiratory disease was also found to be associated with pain/discomfort $(\mathrm{p}=0.001)$ and anxiety/depression $(p=0.012)$. Religion $(p=0.027)$, work type $(p=0.036)$, smokeless tobacco intake $(p=0.004)$, alcohol drinking $(\mathrm{p}=0.009)$ and respiratory disease $(\mathrm{p}=0.001$ ) were found to be associated with the EQ-VAS.

Tables 2 and 3 shows the multiple backward stepwise regression analyses to determine factors independently associated with the EQ-5D dimensions and EQ-VAS. The odds of mobility problems decreased with age (OR 0.95, $95 \%$ CI 0.91 to $0.99, p=0.016$ ), were lower in participants with presence/history of any respiratory disease $(0.27$, 0.13 to $0.55, \mathrm{p}<0.001), \mathrm{SC} / \mathrm{ST} / \mathrm{OBC}$ workers $(0.44,0.22$ to $0.89, \mathrm{p}=0.021)$, manual workers $(0.40,0.16$ to 0.99 , $\mathrm{p}=0.047)$ and non-smokers (2.63, 1.27 to $5.46, \mathrm{p}=0.009$ ). The odds of pain/discomfort and anxiety/depression were lower in participants with any respiratory disease (0.44, 0.24 to $0.79, \mathrm{p}=0.006$; and $0.52,0.29$ to 0.92 , 
Table 1 Characteristics of coal-based sponge-iron factory workers with no problem and problems in the EQ-5D dimensions and EQ-VAS

\begin{tabular}{|c|c|c|c|c|c|c|c|c|c|c|c|c|c|c|c|c|c|}
\hline & \multicolumn{3}{|c|}{ Mobility $(n=257)$} & \multicolumn{3}{|c|}{ Self-care $(n=256)$} & \multicolumn{3}{|c|}{ Usual activities $(n=256$ ) } & \multicolumn{3}{|c|}{ Pain/discomfort $(n=256)$} & \multicolumn{3}{|c|}{ Anxiety/depression $(n=257)$} & \multicolumn{2}{|c|}{ EQ-VAS $(n=256)$} \\
\hline & $\begin{array}{l}\text { No problem } \\
(n=197)\end{array}$ & $\begin{array}{l}\text { Problem } \\
(n=60)\end{array}$ & $\begin{array}{l}p \\
\text { Value }\end{array}$ & $\begin{array}{l}\text { No problem } \\
(n=243)\end{array}$ & $\begin{array}{l}\text { Problem } \\
(n=13)\end{array}$ & $\begin{array}{l}p \\
\text { Value }\end{array}$ & $\begin{array}{l}\text { No problem } \\
(n=228)\end{array}$ & $\begin{array}{l}\text { Problem } \\
(n=28)\end{array}$ & $\begin{array}{l}p \\
\text { Value }\end{array}$ & $\begin{array}{l}\text { No } \\
(n=155)\end{array}$ & $\begin{array}{l}\text { Yes } \\
(n=101)\end{array}$ & $\begin{array}{l}\mathbf{p} \\
\text { Value }\end{array}$ & $\begin{array}{l}\text { No } \\
(n=140)\end{array}$ & $\begin{array}{l}\text { Yes } \\
(n=117)\end{array}$ & $\begin{array}{l}\mathbf{p} \\
\text { Value }\end{array}$ & $\begin{array}{l}\text { Mean } \\
\text { (SD) }\end{array}$ & $\begin{array}{l}\mathbf{p} \\
\text { Value }\end{array}$ \\
\hline Age & $34.9(8.5)^{\star}$ & $37.0(7.0)^{\star}$ & $0.091 \dagger$ & $35.4(8.3)^{\star}$ & $36.6(6.5)^{\star}$ & $0.593 \dagger$ & $35.4(8.5)^{\star}$ & $35.1(5.0)^{\star}$ & $0.835 t$ & $34.8(7.8)^{\star}$ & $\begin{array}{l}36.3 \\
(8.7)^{\star}\end{array}$ & $0.144 \dagger$ & $35.1(8.2)^{\star}$ & $\begin{array}{l}35.8 \\
(8.2)^{*}\end{array}$ & $0.465 \dagger$ & $35.4(8.2)$ & $0.779 \dagger$ \\
\hline Mother tongue & & & 0.695 & & & 1.000 & & & 0.218 & & & 0.521 & & & 0.564 & & 0.855 \\
\hline Bengali & $168(85.3)$ & $53(88.3)$ & & $209(86.0)$ & $12(92.3)$ & & $193(84.6)$ & $27(96.4)$ & & $135(87.1)$ & $85(84.2)$ & & $117(83.6)$ & $\begin{array}{l}104 \\
(88.9)\end{array}$ & & $69.7(18.4)$ & \\
\hline Other & $23(11.7)$ & $6(10.0)$ & & $27(11.1)$ & $1(7.7)$ & & $28(12.3)$ & $1(3.6)$ & & $16(10.3)$ & $13(12.9)$ & & $17(12.1)$ & $12(10.3)$ & & $70.4(20.8)$ & \\
\hline Unknown & $6(3.0)$ & $1(1.7)$ & & $7(2.9)$ & 0 & & $7(3.1)$ & 0 & & $4(2.6)$ & $3(3.0)$ & & $6(4.3)$ & $1(0.9)$ & & $71.4(15.7)$ & \\
\hline Religion & & & 0.289 & & & 0.443 & & & 0.106 & & & 1.000 & & & 0.354 & & 0.027 \\
\hline Hindu & $190(96.4)$ & $56(93.3)$ & & $233(95.9)$ & $12(92.3)$ & & $220(96.5)$ & $25(89.3)$ & & $148(95.5)$ & $97(96.0)$ & & $132(94.3)$ & $\begin{array}{l}114 \\
(97.4)\end{array}$ & & $69.3(18.5)$ & \\
\hline Islam & $7(3.6)$ & $4(6.7)$ & & $10(4.1)$ & $1(7.7)$ & & $8(3.5)$ & $3(10.7)$ & & $7(4.5)$ & $4(4.0)$ & & $8(5.7)$ & $3(2.6)$ & & $81.8(14.7)$ & \\
\hline Social caste & & & 0.146 & & & 0.546 & & & 0.067 & & & 0.893 & & & 0.515 & & 0.898 \\
\hline General & $128(65.0)$ & $33(55.0)$ & & $152(62.6)$ & $9(69.2)$ & & $148(64.9)$ & $13(46.4)$ & & $97(62.6)$ & $64(63.4)$ & & $90(64.3)$ & $71(60.7)$ & & $69.5(19.1)$ & \\
\hline $\mathrm{SC} / \mathrm{ST} / \mathrm{OBC}$ & $65(33.0)$ & $26(43.3)$ & & $87(35.8)$ & $3(23.1)$ & & $76(33.3)$ & $14(50.0)$ & & $55(35.5)$ & $35(34.7)$ & & 47 (33.6) & $44(37.6)$ & & $69.8(17.7)$ & \\
\hline Unknown & $4(2.0)$ & $1(1.7)$ & & $4(1.6)$ & $1(7.7)$ & & $4(1.8)$ & $1(3.6)$ & & $3(1.9)$ & $2(2.0)$ & & $3(2.1)$ & $2(1.7)$ & & $79.0(13.4)$ & \\
\hline Marriage & & & 0.031 & & & 1.000 & & & 0.777 & & & 0.324 & & & 0.448 & & 0.481 \\
\hline Yes & $165(83.8)$ & $55(91.7)$ & & $208(85.6)$ & $11(84.6)$ & & $194(85.1)$ & 25 (89.3) & & $131(84.5)$ & $88(87.1)$ & & $122(87.1)$ & 98 (83.8) & & $69.4(18.1)$ & \\
\hline No & $32(16.2)$ & $3(5.0)$ & & $34(14.0)$ & $1(7.7)$ & & $32(14.0)$ & $3(10.7)$ & & $24(15.5)$ & $11(10.9)$ & & $17(12.1)$ & 18 (15.4) & & $71.8(20.6)$ & \\
\hline Unknown & 0 & $2(3.3)$ & & $1(0.4)$ & $1(7.7)$ & & $2(0.9)$ & 0 & & 0 & $2(2.0)$ & & $1(0.7)$ & $1(0.9)$ & & 75.0 (35.4) & \\
\hline Education & & & 0.306 & & & 1.000 & & & 1.000 & & & 0.913 & & & 0.948 & & 0.119 \\
\hline Literate & $170(86.3)$ & $55(91.7)$ & & $212(87.2)$ & $12(92.3)$ & & $199(87.3)$ & $25(89.3)$ & & $135(87.1)$ & $89(88.1)$ & & $122(87.1)$ & $\begin{array}{l}103 \\
(88.0)\end{array}$ & & $69.0(18.5)$ & \\
\hline Illiterate & 26 (13.2) & $5(8.3)$ & & 30 (12.3) & $1(7.7)$ & & 28 (12.3) & $3(10.7)$ & & $19(12.3)$ & $12(11.9)$ & & $17(12.1)$ & $14(12.0)$ & & $74.5(17.6)$ & \\
\hline Unknown & $1(0.5)$ & 0 & & $1(0.4)$ & 0 & & $1(0.4)$ & 0 & & $1(0.6)$ & 0 & & $1(0.7)$ & 0 & & $100(0)$ & \\
\hline Work type & & & 0.234 & & & 0.495 & & & 0.151 & & & 0.896 & & & 0.536 & & 0.036 \\
\hline Non-manual & 47 (23.9) & $10(16.7)$ & & $53(21.8)$ & $4(30.8)$ & & $54(23.7)$ & $3(10.7)$ & & $34(21.9)$ & $23(22.8)$ & & 33 (23.6) & $24(20.5)$ & & $74.2(19.1)$ & \\
\hline Manual & $149(75.6)$ & 50 (83.3) & & $189(77.8)$ & $9(69.2)$ & & $173(75.9)$ & $25(89.3)$ & & $120(77.4)$ & 78 (77.2) & & $106(75.7)$ & 93 (79.5) & & $68.4(18.1)$ & \\
\hline Unknown & $1(0.5)$ & 0 & & $1(0.4)$ & 0 & & $1(0.4)$ & 0 & & $1(0.6)$ & 0 & & $1(0.7)$ & 0 & & $100(0)$ & \\
\hline Working hours/day & & & 0.020 & & & 1.000 & & & 1.000 & & & 0.609 & & & 0.808 & & 0.302 \\
\hline 8 & $173(87.8)$ & $59(98.3)$ & & $219(90.1)$ & $12(92.3)$ & & 205 (89.9) & 26 (92.9) & & $138(89.0)$ & $93(92.1)$ & & $125(89.3)$ & $\begin{array}{l}107 \\
(91.5)\end{array}$ & & $69.3(18.3)$ & \\
\hline$>8$ & $22(11.2)$ & $1(1.7)$ & & $22(9.1)$ & $1(7.7)$ & & $21(9.2)$ & $2(7.1)$ & & $15(9.7)$ & $8(7.9)$ & & $13(9.3)$ & $10(8.5)$ & & $73.5(20.0)$ & \\
\hline Unknown & $2(1.0)$ & 0 & & $2(0.8)$ & 0 & & $2(0.9)$ & 0 & & $2(1.3)$ & 0 & & $2(1.4)$ & 0 & & $85.0(21.2)$ & \\
\hline Salary/month & & & 0.829 & & & 0.524 & & & 0.173 & & & 0.677 & & & 0.786 & & 0.451 \\
\hline$\geq 5395$ INR & $52(26.4)$ & $15(25.0)$ & & $65(26.7)$ & $2(15.4)$ & & $62(27.2)$ & $4(14.3)$ & & $42(27.1)$ & $25(24.8)$ & & $38(27.1)$ & $30(25.6)$ & & $71.3(18.0)$ & \\
\hline$<5395$ INR & $145(73.6)$ & $45(75.0)$ & & $178(73.3)$ & $11(84.6)$ & & $166(72.8)$ & $24(85.7)$ & & $113(72.9)$ & $76(75.2)$ & & $102(72.9)$ & $87(74.4)$ & & $69.3(18.7)$ & \\
\hline Total duration of work & $9.1(11.6)^{*}$ & $11.6(16.5)^{\star}$ & $0.031 \dagger$ & $9.8(13.3)^{*}$ & $7.9(2.8)^{\star}$ & $0.990 \dagger$ & $9.8(13.7)^{\star}$ & $8.4(1.6)^{\star}$ & $0.307 \dagger$ & $8.9(10.8)^{*}$ & $\begin{array}{l}10.8 \\
(15.7)^{\star}\end{array}$ & $0.332 \dagger$ & $8.7(8.3)^{\star}$ & $\begin{array}{l}10.9 \\
(16.8)^{*}\end{array}$ & $0.423 \dagger$ & $10.0(14.1)$ & $0.124 \dagger$ \\
\hline Smoking & & & 0.060 & & & 0.771 & & & 0.442 & & & 0.565 & & & 0.478 & & 0.746 \\
\hline No & $114(57.9)$ & $43(71.7)$ & & $148(60.9)$ & $9(69.2)$ & & $137(60.1)$ & $19(67.9)$ & & $97(62.6)$ & $60(59.4)$ & & $88(62.9)$ & $69(59.0)$ & & $69.4(18.6)$ & \\
\hline Yes & $82(41.6)$ & $17(28.3)$ & & $94(38.7)$ & $4(30.8)$ & & $90(39.5)$ & $9(32.1)$ & & $57(36.8)$ & $41(40.6)$ & & $51(36.4)$ & $48(41.0)$ & & $70.2(18.3)$ & \\
\hline Unknown & $1(0.5)$ & 0 & & $1(0.4)$ & 0 & & $1(0.4)$ & 0 & & $1(0.6)$ & 0 & & $1(0.7)$ & 0 & & $100(0)$ & \\
\hline $\begin{array}{l}\text { Smokeless } \\
\text { tobacco intake }\end{array}$ & & & 0.414 & & & 0.765 & & & 0.104 & & & 0.317 & & & 0.900 & & 0.004 \\
\hline No & $100(50.8)$ & $27(45.0)$ & & $120(55.8)$ & $7(53.8)$ & & $109(47.8)$ & $18(64.3)$ & & $80(51.6)$ & $46(45.5)$ & & $70(50.0)$ & $58(49.6)$ & & $73.0(18.3)$ & \\
\hline Yes & $96(48.7)$ & $33(55.0)$ & & $122(43.7)$ & $6(46.2)$ & & $118(51.8)$ & $10(35.7)$ & & $74(47.7)$ & 55 (54.5) & & 69 (49.3) & $59(50.4)$ & & $66.4(18.1)$ & \\
\hline
\end{tabular}




\begin{tabular}{|c|c|c|c|c|c|c|c|c|c|c|c|c|c|c|c|c|c|}
\hline & \multicolumn{3}{|c|}{ Mobility $(n=257)$} & \multicolumn{3}{|c|}{ Self-care $(n=256)$} & \multicolumn{3}{|c|}{ Usual activities $(n=256)$} & \multicolumn{3}{|c|}{ Pain/discomfort $(n=256)$} & \multicolumn{3}{|c|}{ Anxiety/depression ( $n=257)$} & \multicolumn{2}{|c|}{ EQ-VAS $(n=256)$} \\
\hline & $\begin{array}{l}\text { No problem } \\
(n=197)\end{array}$ & $\begin{array}{l}\text { Problem } \\
(n=60)\end{array}$ & $\begin{array}{l}p \\
\text { Value }\end{array}$ & $\begin{array}{l}\text { No problem } \\
(n=243)\end{array}$ & $\begin{array}{l}\text { Problem } \\
(n=13)\end{array}$ & $\begin{array}{l}p \\
\text { Value }\end{array}$ & $\begin{array}{l}\text { No problem } \\
(n=228)\end{array}$ & $\begin{array}{l}\text { Problem } \\
(n=28)\end{array}$ & $\begin{array}{l}p \\
\text { Value }\end{array}$ & $\begin{array}{l}\text { No } \\
(n=155)\end{array}$ & $\begin{array}{l}\text { Yes } \\
(n=101)\end{array}$ & $\begin{array}{l}p \\
\text { Value }\end{array}$ & $\begin{array}{l}\text { No } \\
(n=140)\end{array}$ & $\begin{array}{l}\text { Yes } \\
(n=117)\end{array}$ & $\begin{array}{l}p \\
\text { Value }\end{array}$ & $\begin{array}{l}\text { Mean } \\
\text { (SD) }\end{array}$ & $\begin{array}{l}p \\
\text { Value }\end{array}$ \\
\hline Unknown & $1(0.5)$ & 0 & & $1(0.5)$ & 0 & & $1(0.4)$ & 0 & & $1(0.6)$ & 0 & & $1(0.7)$ & 0 & & $100(0)$ & \\
\hline Alcohol drinking & & & 0.754 & & & 1.000 & & & 0.875 & & & 0.092 & & & 0.062 & & 0.009 \\
\hline No & $129(65.5)$ & $41(68.3)$ & & $161(66.3)$ & $9(69.2)$ & & $150(65.8)$ & $19(67.9)$ & & $108(69.7)$ & $61(60.4)$ & & $99(70.7)$ & $71(60.7)$ & & $71.7(19.0)$ & \\
\hline Yes & $66(33.5)$ & $19(31.7)$ & & $80(32.9)$ & $4(30.8)$ & & 76 (33.3) & $9(32.1)$ & & $45(29.0)$ & 40 (39.6) & & 39 (27.9) & 46 (39.3) & & $65.4(16.6)$ & \\
\hline Unknown & $2(1.0)$ & 0 & & $2(0.8)$ & 0 & & $2(0.9)$ & 0 & & $2(1.3)$ & 0 & & $2(1.4)$ & 0 & & $92.5(10.6)$ & \\
\hline House type & & & 0.395 & & & 0.798 & & & 0.423 & & & 0.369 & & & 0.500 & & 0.573 \\
\hline Pucca/semipucca & $100(50.8)$ & $27(45.0)$ & & $120(49.4)$ & $6(46.2)$ & & $115(50.4)$ & $12(42.9)$ & & $73(47.1)$ & $54(53.5)$ & & $66(47.1)$ & $60(51.3)$ & & $70.4(17.9)$ & \\
\hline Kachha & $95(48.2)$ & $33(55.0)$ & & $121(49.8)$ & 7 (53.8) & & $111(48.7)$ & $16(57.1)$ & & $80(51.6)$ & $47(46.5)$ & & $73(52.1)$ & $56(47.9)$ & & $69.1(19.2)$ & \\
\hline Unknown & $2(1.0)$ & 0 & & $2(0.8)$ & 0 & & $2(0.9)$ & 0 & & $2(1.3)$ & 0 & & $1(0.7)$ & $1(0.9)$ & & $75.0(14.1)$ & \\
\hline People in a room & & & 0.071 & & & 0.760 & & & 0.746 & & & 0.666 & & & 0.973 & & 0.825 \\
\hline$<3$ & $67(34.0)$ & $13(21.7)$ & & $77(31.7)$ & $3(23.1)$ & & $72(31.6)$ & $8(28.6)$ & & $50(32.3)$ & $30(29.7)$ & & $44(31.4)$ & 37 (31.6) & & $69.4(18.9)$ & \\
\hline$\geq 3$ & $130(66.0)$ & 47 (78.3) & & $166(68.3)$ & $10(76.9)$ & & $156(68.4)$ & $20(71.4)$ & & $105(67.7)$ & 71 (70.3) & & 96 (68.6) & 80 (68.4) & & $70.0(18.4)$ & \\
\hline $\begin{array}{l}\text { Presence/history of } \\
\text { respiratory disease }\end{array}$ & & & $<0.001$ & & & 0.065 & & & 0.427 & & & 0.001 & & & 0.012 & & 0.001 \\
\hline No & $150(76.1)$ & $30(50.0)$ & & $173(71.2)$ & $6(46.2)$ & & $161(70.6)$ & $18(64.3)$ & & $121(78.1)$ & $59(58.4)$ & & $106(75.7)$ & $74(63.2)$ & & $71.9(18.9)$ & \\
\hline Yes & $41(20.8)$ & $25(41.7)$ & & $60(24.7)$ & $6(46.2)$ & & $57(25.0)$ & $9(32.1)$ & & $29(18.7)$ & 36 (35.6) & & $27(19.3)$ & 39 (33.3) & & $63.1(16.0)$ & \\
\hline Unknown & $6(3.0)$ & $5(8.3)$ & & $10(4.1)$ & $1(7.7)$ & & $10(4.4)$ & $1(3.6)$ & & $5(3.2)$ & $6(5.9)$ & & $7(5.0)$ & $4(3.4)$ & & $75.9(16.9)$ & \\
\hline Health insurance & & & 0.197 & & & 0.719 & & & 0.616 & & & 0.588 & & & 0.922 & & 0.964 \\
\hline No & $41(20.8)$ & $8(13.3)$ & & $46(18.9)$ & $3(23.1)$ & & $45(19.7)$ & $4(14.3)$ & & $28(18.1)$ & $21(20.8)$ & & $27(19.3)$ & $22(18.8)$ & & $69.7(20.1)$ & \\
\hline Yes & $156(79.2)$ & $52(86.7)$ & & $197(81.1)$ & $10(76.9)$ & & $183(80.3)$ & $24(85.7)$ & & $127(81.9)$ & $80(79.2)$ & & $113(80.7)$ & $95(81.2)$ & & $69.8(18.2)$ & \\
\hline
\end{tabular}


Table 2 Multiple backward stepwise regression analyses to determine factors independently associated with the EQ-5D dimensions

\begin{tabular}{|c|c|c|c|}
\hline & OR & $95 \% \mathrm{Cl}$ & p Value \\
\hline \multicolumn{4}{|l|}{ Mobility problem } \\
\hline Age & 0.95 & 0.91 to 0.99 & 0.016 \\
\hline \multicolumn{4}{|c|}{ Presence/history of respiratory disease } \\
\hline No & 1 & & $<0.001$ \\
\hline Yes & 0.27 & 0.13 to 0.55 & \\
\hline \multicolumn{4}{|l|}{ Smoking } \\
\hline No & 1 & & 0.009 \\
\hline Yes & 2.63 & 1.27 to 5.46 & \\
\hline \multicolumn{4}{|l|}{ Social caste } \\
\hline General & 1 & & 0.021 \\
\hline SC/ST/OBC & 0.44 & 0.22 to 0.89 & \\
\hline \multicolumn{4}{|c|}{ Working hours/day } \\
\hline 8 & 1 & & 0.065 \\
\hline$>8$ & 7.35 & 0.88 to 61.07 & \\
\hline \multicolumn{4}{|l|}{ Education } \\
\hline Literate & 1 & & 0.089 \\
\hline Illiterate & 2.64 & 0.86 to 8.08 & \\
\hline \multicolumn{4}{|l|}{ Work type } \\
\hline Non-manual & 1 & & 0.047 \\
\hline Manual & 0.40 & 0.16 to 0.99 & \\
\hline \multicolumn{4}{|l|}{ Pain/discomfort } \\
\hline \multicolumn{4}{|c|}{ Presence/history of respiratory disease } \\
\hline No & 1 & & 0.006 \\
\hline Yes & 0.44 & 0.24 to 0.79 & \\
\hline \multicolumn{4}{|c|}{ Anxiety/depression } \\
\hline \multicolumn{4}{|c|}{ Alcohol drinking } \\
\hline No & 1 & & 0.076 \\
\hline Yes & 0.62 & 0.36 to 1.05 & \\
\hline \multicolumn{4}{|c|}{ Presence/history of respiratory disease } \\
\hline No & 1 & & 0.026 \\
\hline Yes & 0.52 & 0.29 to 0.92 & \\
\hline
\end{tabular}

$\mathrm{p}=0.026$, respectively). The EQ-VAS was worse in manual participants (coefficient $-6.91,95 \%$ CI -12.40 to -1.41 , $\mathrm{p}=0.014)$, with any respiratory disease $(-8.13,-13.12$ to -3.13, $\mathrm{p}=0.002)$, alcohol drinkers $(-4.81,-9.47$ to $-0.15, \mathrm{p}=0.043)$, literates $(7.70,0.97$ to $14.43, \mathrm{p}=0.025)$ and Hindus (13.41, 2.62 to $24.20, \mathrm{p}=0.015)$.

\section{DISCUSSION}

The percentage of participants with problems in mobility, self-care, usual activities, pain/discomfort and anxiety/ depression were $23.3 \%, 5.1 \%, 10.9 \%, 39.5 \%$ and $45.5 \%$, respectively. This indicates that in spite of the presence of anxiety/depression, pain/discomfort and mobility problems, participants were left with no other option than to perform their own self-care and usual activities. The presence of anxiety/depression among participants also indicates their poor mental health status. In China, a neighbouring country with a similar fast-growing economy, political transition, large population and
Table 3 Multiple backward stepwise regression analyses to determine factors independently associated with the EQ-VAS

\begin{tabular}{|c|c|c|c|}
\hline & Coefficient & $95 \% \mathrm{Cl}$ & p Value \\
\hline \multicolumn{4}{|l|}{ Alcohol drinking } \\
\hline No & 0 & & 0.043 \\
\hline Yes & -4.81 & -9.47 to -0.15 & \\
\hline \multicolumn{4}{|l|}{ Work type } \\
\hline Non-manual & 0 & & 0.014 \\
\hline Manual & -6.91 & -12.40 to -1.41 & \\
\hline \multicolumn{4}{|l|}{ Education } \\
\hline Literate & 0 & & 0.025 \\
\hline Illiterate & 7.70 & 0.97 to 14.43 & \\
\hline \multicolumn{4}{|l|}{ Religion } \\
\hline Hindu & 0 & & 0.015 \\
\hline Islam & 13.41 & 2.62 to 24.20 & \\
\hline \multicolumn{4}{|c|}{$\begin{array}{l}\text { Presence/history of } \\
\text { respiratory disease }\end{array}$} \\
\hline No & 0 & & 0.002 \\
\hline Yes & -8.13 & -13.12 to -3.13 & \\
\hline
\end{tabular}

sociohealth inequalities, reported problems in EQ-5D dimensions were found to be much lower in the general population (corresponding figures 4.3\%, $2.7 \%, 4 \%, 7.2 \%$ and $5.2 \%$ of men). ${ }^{8}$ Our findings are similar to the UK (corresponding figures 18.4\%, 4.2\%, 16.3\%, 33\% and $20.9 \%$ of the general population). ${ }^{9}$ Although India and the UK are different in many terms including economy, population and healthcare system, they are bound by strong ties of shared history and culture which could partially explain this similarity. Health state 11111 was reported by only $36.8 \%$ of participants. This proportion was much lower compared with the Chinese study $(87 \%)$, but was similar to the UK study $(36 \%) .{ }^{89}$ The Cronbach's $\alpha$ of EQ-5D dimensions was 0.75 , which indicates good internal consistency reliability. The mean EQ-VAS was 69.8 (18.5 SD), whereas it was 80.9 and 82.5 in China and the UK, respectively. ${ }^{8}{ }^{9}$ A study conducted among aluminium-smelting factory workers in Norway also reported poor HRQoL among workers. ${ }^{14}$

In the unadjusted models, marriage, working hours/ day, total duration of work in this type of factory and presence/history of any respiratory disease were found to be associated with mobility. Respiratory disease was also found to be associated with pain/discomfort and anxiety/depression. Religion, work type, smokeless tobacco intake, alcohol drinking and respiratory disease were found to be associated with the EQ-VAS. Previous studies conducted among the general population and other workforce groups in various countries reported similar and other factors associated with HRQoL (such as age, education, social status, marital status, smoking, alcohol drinking, work type, working hours, occupation, salary, disease and health insurance). ${ }^{8-13}$ 
The odds of mobility problems in our study decreased with age. This could be explained by the fact that there is an increasing global trend of sedentary lifestyle (and diet) among younger people, ${ }^{22}$ which has a negative implication on physical fitness, and mobility problems are becoming common in middle-aged adults. ${ }^{23}$ However, problems reported in the EQ-5D dimensions increased with age in China and the UK (except anxiety/depression), and the EQ-VAS also decreased with age. ${ }^{8}{ }^{9}$ The odds of mobility problems, pain/discomfort and anxiety/depression were lower in participants with presence/history of any respiratory disease as compared with those with no such issue. Intuitively, one would expect the opposite and this issue requires further investigation. However, as one would expect they had a worse EQ-VAS as compared with those with no such issue. This is consistent with another study conducted among the same population, where respiratory disease was found to be associated with worse respiratory HRQoL. ${ }^{24}$ The odds of mobility problems were lower in $\mathrm{SC} / \mathrm{ST} / \mathrm{OBC}$ and manual participants. In general, SC/ ST/OBC people are largely involved in manual work, and the physical work and active lifestyle may be keeping them fit (in terms of mobility) in this current global trend towards a sedentary lifestyle. This is consistent with another study conducted among the same population, where manual work was found to be associated with better respiratory HRQoL in terms of activity. ${ }^{24}$ Smokers had increased odds of mobility problems as compared with non-smokers. A study conducted among blue-collar and white-collar industrial workers in Greece also reported the negative impact of smoking on mobility along with that on self-care, anxiety/depression and the EQ-VAS. ${ }^{10}$ The UK study also reported the negative impact of smoking on all the EQ-5D dimensions and EQ-VAS. ${ }^{9}$

Manual participants and alcohol drinkers had worse EQ-VAS. The second association is consistent with another study conducted among the same population, where alcohol drinking was found to be associated with worse respiratory HRQoL. ${ }^{24}$ Intuitively, one would expect illiterates to have worse EQ-VAS than literates, and Muslims and Hindus to have similar EQ-VAS. However, literates and Hindus had worse EQ-VAS as compared with illiterates and Muslims, respectively, reflecting one of the definitions of HRQoL as the gap between expectations of health and the experience of it. ${ }^{25}$ This finding could be due to lower expectations of health among illiterates and Muslims, similar to people with lower socioeconomic status who might rate their own health status higher than people with higher socioeconomic status. ${ }^{26}{ }^{27}$ However, the EQ-VAS was significantly lower in people with lower levels of education (in China and the UK), and in lower income groups (in China). ${ }^{8}{ }^{9}$ A study of Greek industrial workers also reported education as a significant predictor of EQ-VAS. ${ }^{10}$ In this study, illiterate participants may have reported better health status to field workers (who completed the questionnaire on their behalf) compared with self-completion by literate participants. In some studies, participants reported better health status during face-to-face interviews than in postal surveys. ${ }^{28-31}$ This issue requires further exploration.

This study has a number of strengths and weaknesses. As far as we are aware, this is the first study to explore HRQoL of coal-based sponge iron plant workers. All the workers who were approached to participate in the study responded $(100 \%$ response rate and thus, no nonrespondents). This indicates that the data collection methodology was appropriate and there is more certainty in the study findings (ie, it is more likely the results are representative of the population). In terms of generalisability, the study findings could be valid in settings with similar populations and healthcare systems (such as in other South-Asian countries). The standard steps in questionnaire development (design, translation and pretesting) were followed to ensure the validity and reliability of the questionnaire (Section I). HRQoL measurement is subjective to participants and thus, a valid and reliable tool (EQ-5D-5L in English, Bengali and Hindi) was used. The field workers used a standardised protocol for data collection. The field workers and the participants belonged to the same culture, which minimised the scope for cultural bias in the study. Missing data could lead to bias, but it was extremely low in this study. Multiple regression analyses included a sample with missing values for the adjusted variables. Participants who were absent from work on the dates of the survey were excluded, and this absence from work could be due to poor HRQoL which could have underestimated the prevalence of poor HRQoL. Most of the data were self-reported, and recall error could have been a problem. Medical records might be a more reliable measure (for the presence/history of any respiratory disease), but these were not available/accessible in the study area. Lung function or other diagnostic tests could have been used, but the aim of the study was to explore workers' perception of health (HRQoL). However, these tests could be used in future studies, which would crosscheck our study findings and would provide a complete picture of the scenario. Several associations that were found in the study deserve further examination, and not just explaining the association based on assumptions. For example, the odds of mobility problems decreased with participants' age. It is possible that these findings were the result of other confounding factors not adjusted for in the models. The aim of the study was to explore these workers' HRQoL, and there was no control group in the study. The study findings are compared with studies conducted among the general population and other workforce groups in various countries, ${ }^{8-14}$ as HRQoL studies conducted among coalbased sponge iron plant workers are lacking. Thus, similar research needs to be conducted among coalbased sponge iron plant employees working in other parts of India and other countries to enhance the 
generalisability of these results. As this was a crosssectional study, it was not possible to determine the causal association between different variables and HRQoL. A long-term, longitudinal study should be conducted among these coal-based sponge iron factory workers to assess the impact of various factors (these as well as other potential factors) on HRQoL. A good example would be to have a cohort study comparing coal-based sponge iron factory workers with other types of factory workers (healthy worker effect), rather than with the general population.

In conclusion, many coal-based sponge iron plant workers in Barjora report problems in their HRQoL, and the predictors of different aspects of HRQoL were identified. The study findings could be taken into consideration in future interventional studies aimed at improving the HRQoL of these workers. Some of the associated factors are hard to change (such as work type and presence/history of any respiratory disease), while some of them (lifestyle and behavioural factors such as smoking and alcohol) are easier to prevent or change.

Acknowledgements The authors thank the funding agency, Suresh Chandra Chattopadhyay, field workers, Barjora BD0, factory directors and the participants.

Contributors KC designed the study. KC, CC and EK conducted the study. KC wrote the first draft of the manuscript. All authors revised for important intellectual content and approved the final manuscript.

Funding This study was funded by a grant from the Blacksmith Institute, USA

\section{Competing interests None.}

Patient consent Obtained

Ethics approval Barjora Block Development Office Committee.

Provenance and peer review Not commissioned; externally peer reviewed.

Data sharing statement No additional data are available.

Open Access This is an Open Access article distributed in accordance with the Creative Commons Attribution Non Commercial (CC BY-NC 4.0) license, which permits others to distribute, remix, adapt, build upon this work noncommercially, and license their derivative works on different terms, provided the original work is properly cited and the use is non-commercial. See: http:// creativecommons.org/licenses/by-nc/4.0/

\section{REFERENCES}

1. Patra HS, Sahoo B, Mishra BK. Status of sponge iron plants in Orissa. Bhubaneswar, India: Vasundhara, 2012.

2. Centre for Science and Environment (CSE). Sponge iron industry: the regulatory challenge. New Delhi, India: CSE, 2011.

3. Chatterjee S. Source, dispersal and impacts of airborne pollutants: a case study of Mangalpur industrial complex, Raniganj. J Hum Ecol 2011;35:195-201.

4. Cerana Foundation. Risk appraisal study: sponge iron plants, Raigarh district. Hyderabad, India: Cerana Foundation, 2006.

5. Institute of Medicine. Crossing the quality chasm: a new health system for the 21st century. Washington, DC: National Academies Press, 2001.

6. Fayers P, Machin D. Quality of life: the assessment, analysis and interpretation of patient-reported outcomes. 2nd edn. Chichester, UK: John Wiley and Sons, 2007.
7. World Health Organization. Study protocol for the World Health Organization project to develop a quality of life assessment instrument (WHOQOL). Qual Life Res 1993;2:153-9.

8. Sun $\mathrm{S}$, Chen J, Johannesson M, et al. Population health status in China: EQ-5D results, by age, sex and socio-economic status, from the National Health Services Survey 2008. Qual Life Res 2011;20:309-20.

9. Kind $\mathrm{P}$, Dolan $\mathrm{P}$, Gudex $\mathrm{C}$, et al. Variations in population health status: results from a United Kingdom national questionnaire survey. BMJ 1998;316:736-41.

10. Rachiotis G, Behrakis PK, Vasiliou M, et al. Quality of life and smoking among industrial workers in Greece. Med Lav 2006;97:44-50.

11. Caban-Martinez AJ, Lee DJ, Fleming LE, et al. Arthritis, occupational class, and the aging US workforce. Am J Public Health 2011;101:1729-34.

12. Zhu C, Geng Q, Yang H, et al. Quality of life in China rural-to-urban female migrant factory workers: a before-and-after study. Health Qual Life Outcomes 2013;11:123.

13. Wu SY, Li HY, Tian J, et al. Health-related quality of life and its main related factors among nurses in China. Ind Health 2011;49: 158-65.

14. Morken T, Riise T, Moen B, et al. Frequent musculoskeletal symptoms and reduced health-related quality of life among industrial workers. Occup Med (Lond) 2002;52:91-8.

15. Office of the Labour Commissioner (OLC), Government of West Bengal. Monthly minimum rates of wages. Kolkata, India: OLC, 2013.

16. Mishra VK, Retherford RD, Smith KR. Biomass cooking fuels and prevalence of blindness in India. J Environ Med 1999;1: 189-99.

17. Rabin $\mathrm{R}$, Oemar $\mathrm{M}$, Oppe $\mathrm{M}$, et al. EQ-5D-5L user guide: basic information on how to use the EQ-5D-5L instrument, version 1.0. Rotterdam, the Netherlands: EuroQol Group.

18. Creative Research Systems (CRS). Sample size calculator. California: CRS, 2012. http://www.surveysystem.com/sscalc.htm (accessed 1 May 2013)

19. Urbaniak GC, Plous S. Research randomizer (version 4.0) [computer software]. Social Psychology Network. 2013. http://www. randomizer.org/form.htm (accessed 1 May 2013).

20. Indian Council of Medical Research (ICMR). ICMR ethical guidelines for biomedical research on human participants. Delhi, India: ICMR, 2006.

21. STATACorp. STATA statistical software: release 12. College Station. TX: STATACorp LP, 2011

22. Ding D, Sallis JF, Hovell MF, et al. Physical activity and sedentary behaviours among rural adults in Suixi, China: a cross-sectional study. Int J Behav Nutr Phys Act 2011;8:37.

23. Lezzoni LI, McCarthy EP, Davis RB, et al. Mobility difficulties are not only a problem of old age. J Gen Intern Med 2001;16: 235-43.

24. Chattopadhyay K, Chattopadhyay C, Kaltenthaler E. Respiratory health-related quality-of-life of coal-based sponge iron plant workers in Barjora, India: a cross-sectional study. BMJ Open Resp Res Submitted.

25. Carr AJ, Gibson B, Robinson PG. Is quality of life determined by expectations or experience? BMJ 2001;322:1240-3.

26. Sen A. Health: perception versus observation: self reported morbidity has severe limitations and can be extremely misleading. BMJ 2002;324:860-1.

27. Symon Z, Daignault S, Symon R, et al. Measuring patients' expectations regarding health-related quality-of-life outcomes associated with prostate cancer surgery or radiotherapy. Urology 2006;68:1224-9.

28. Bowling A, Bond M, Jenkinson C, et al. Short Form 36 (SF-36) Health Survey questionnaire: which normative data should be used? Comparisons between the norms provided by the Omnibus Survey in Britain, the Health Survey for England and the Oxford Healthy Life Survey. J Public Health Med 1999;21:255-70.

29. Weinberger M, Oddone EZ, Samsa GP, et al. Are health-related quality-of-life measures affected by the mode of administration? $J$ Clin Epidemiol 1996;49:135-40.

30. Bowling A. Mode of questionnaire administration can have serious effects on data quality. J Public Health (Oxf) 2005;27:281-91.

31. Norman R, King MT, Clarke D, et al. Does mode of administration matter? Comparison of online and face-to-face administration of a time trade-off task. Qual Life Res 2010;19:499-508. 